International Journal of Difference Equations (IJDE).

ISSN 0973-6069, Volume 16, Number 1, (2021). 123-135

(C) Research India Publications

https://dx.doi.org/10.37622/IJDE/16.1.2021.123-135

\title{
New Oscillation Conditions for Second-Order Delay Difference Equations with Several Sub-Linear Neutral Terms
}

\author{
C. Soundara Rajan* ${ }^{*}$ A. Murugesan ${ }^{\dagger}$, and P. Gopalakrishnan ${ }^{\ddagger}$ \\ *Department of Mathematics, Government Arts College (Autonomous), Salem - \\ 636007, Tamil Nadu, India. Corresponding author \\ ${ }^{\dagger}$ Department of Mathematics, Government Arts College (Autonomous), Salem - \\ 636007, Tamil Nadu, India. \\ ${ }^{\ddagger}$ Department of Mathematics, Mahendra Arts \& Science College (Autonomous), \\ Kalipatti, Namakkal Dt., Tamil Nadu, India.
}

\begin{abstract}
We derive oscillatory conditions for the second-order delay difference equation

$$
\Delta\left(\varphi(\zeta)(\Delta v(\zeta))^{\xi}\right)+\mu(\zeta) x^{\nu}(\eta(\zeta))=0 ; \quad \zeta \geq \zeta_{0},
$$

where $v(\zeta)=x(\zeta)+\sum_{i=1}^{m} p_{i}(\zeta) x^{\lambda_{i}}\left(\kappa_{i}(\zeta)\right)$. We investigate oscillatory behavior for the cases when $\xi>\nu$ and $\xi<\nu$. Many results presented in the literature are supplemented and improved by this new theorem. Finally, we give some examples to show our major findings.
\end{abstract}

2020 Mathematics Subject Classifications: 39A12, 39A13, 39A21.

Keywords and Phrases: Oscillation, non-oscillation, second-order, delay, neutral, half-linear, difference equations.

\section{INTRODUCTION}

In this article, we consider the neutral difference equation of the form

$$
\Delta\left(\varphi(\zeta)(\Delta v(\zeta))^{\xi}\right)+\mu(\zeta) x^{\nu}(\eta(\zeta))=0, \quad \zeta \geq \zeta_{0},
$$

\footnotetext{
*Corresponding Author.
} 
where $v(\zeta)=x(\zeta)+\sum_{i=1}^{m} p_{i} x^{\lambda_{i}}\left(\kappa_{i}(\zeta)\right)$. Here $\Delta$ is the forward difference operator given by $\Delta x(\zeta)=x(\zeta+1)-x(\zeta)$. We will assume throughout this work that:

$\left(A_{1}\right) \lambda_{1}, \lambda_{2}, \ldots, \lambda_{m}, \nu, \xi \in\left\{\frac{a}{b}: \mathrm{a}\right.$ and $\mathrm{b}$ are odd positive integers $\}$;

$\left(A_{2}\right)\left\{\kappa_{i}(\zeta)\right\}_{\zeta=\zeta_{0}}^{\infty}(i=1,2, \ldots, m)$ and $\{\eta(\zeta)\}_{\zeta=\zeta_{0}}^{\infty}$ are sequences of positive integers with $\kappa_{i}(\zeta) \leq \zeta(i=1,2, \ldots, m), \eta(\zeta) \leq \zeta, \lim _{\zeta \rightarrow \infty} \kappa_{i}(\zeta)=\lim _{\zeta \rightarrow \infty} \eta(\zeta)=\infty$, $i=1,2, \ldots, m$ and $\Delta \eta(\zeta) \geq 0$;

$\left(A_{3}\right)\{\varphi(\zeta)\}_{\zeta=\zeta_{0}}^{\infty}$ is a sequence of positive real numbers;

$\left(A_{4}\right)\{\mu(\zeta)\}_{\zeta=\zeta_{0}}^{\infty}$ is a sequence of non-negative real numbers and $\mu(\zeta) \not \equiv 0$ for sufficiently large $\zeta$;

$\left(A_{5}\right) \lim _{\zeta \rightarrow \infty} R(\zeta)=\infty$, where $R(\zeta)=\sum_{s=\zeta_{0}}^{\zeta-1} \frac{1}{\varphi^{\frac{1}{\xi}}(s)} ;$

$\left(A_{6}\right)\left\{p_{i}(\zeta)\right\}_{\zeta=\zeta_{0}}^{\infty}$ are positive real sequences for $i=1,2, \ldots, m$.

A solution of (1.1) is said to be oscillatory if its terms are neither eventually positive nor eventually negative; otherwise, it is said to be a non-oscillatory solution. Equation (1.1) is called oscillatory if and only if every solution is oscillatory; otherwise, nonoscillatory.

The existence of solutions, asymptotic behavior, oscillation and non-oscillation for second-order difference equations were extensively analyzed in many research papers over the last three decades; see, for example, $[4,7,8,9,10,11,17,18,20]$ and the cited references. Many areas of applied mathematics use neutral difference and differential equations, such as bifurcation analysis [5], stability theory [22, 23], the dynamical behavior of delayed network systems [24], circuit theory [6], population dynamics [14] and so on. As a result, these equations have piqued people's interest in recent decades. We can refer the monographs $[1,2,3,15,19]$ for the general concept of difference equations.

We [21] determined oscillatory conditions for the second-order half-linear difference equation of advanced type

$$
\Delta\left(\varphi(\zeta)(\Delta x(\zeta))^{\lambda}\right)+\mu(\zeta) x^{\lambda}(\zeta+\eta)=0, \quad \zeta \geq \zeta_{0},
$$

under the condition that $\sum_{\zeta=\zeta_{0}}^{\infty} \frac{1}{\varphi^{\frac{1}{\lambda}}(\zeta)}<\infty$. 
Gopalakrishnan et al. [12] studied oscillatory properties for the non-canonical secondorder difference equation of the delay and advanced type

$$
\Delta(\varphi(\zeta) \Delta x(\zeta))+\mu(\zeta) x(\zeta+\eta)=0 ; \quad \zeta \geq \zeta_{0}
$$

Gopalakrishnan et al. [13] established single-condition criteria for the second-order half-linear advanced difference equation of non-canonical type

$$
\Delta\left(\varphi(\zeta)(\Delta x(\zeta))^{\lambda}\right)+\mu(\zeta) x^{\lambda}(\zeta+\eta)=0, \quad \zeta \geq \zeta_{0}
$$

Our aim in this work is to derive oscillatory condition of all solutions of the equation (1.1) under the cases $\xi>\nu$ and $\xi<\nu$.

In the following sections, we presume that all functional inequalities are satisfied; eventually, that is, for all $\zeta$ large enough.

\section{PRELIMINARY RESULTS}

We use the following notations, for any positive decreasing sequence $\{\psi(\zeta)\}_{\zeta=\zeta_{0}}^{\infty}$ which is converges to zero:

$$
\begin{aligned}
P(\zeta)=\left(1-\sum_{i=1}^{m} \lambda_{i} p_{i}(\zeta)-\frac{1}{\psi(\zeta)} \sum_{i=1}^{m}\left(1-\lambda_{i}\right) p_{i}(\zeta)\right) \geq 0 \\
Q_{1}(\zeta)=\mu(\zeta) P^{\nu}(\eta(\zeta)) \\
Q_{2}(\zeta)=\mu(\zeta) P^{\nu}(\eta(\zeta)) \psi^{\nu-1}(\eta(\zeta)) \\
Q_{3}(\zeta)=\mu(\zeta) P^{\nu}(\eta(\zeta)) R^{\nu-1}\left(\eta(\zeta), \zeta_{1}\right) \\
Q_{4}(\zeta)=\mu(\zeta) P^{\nu}(\eta(\zeta)) R^{\nu}\left(\eta(\zeta), \zeta_{1}\right) \\
U(\zeta)=\sum_{s=\zeta}^{\infty} \mu(s) x^{\nu}(\eta(s)) \geq 0
\end{aligned}
$$

The following lemmas are very useful in proving our results.

Lemma 2.1. [16] Let $a$ and $b$ be nonnegative real numbers. Then

$$
a^{\lambda} b^{1-\lambda} \leq \lambda a+(1-\lambda) \text { b for } o<\lambda \leq 1 .
$$

The equality holds if and only if $a=b$.

Lemma 2.2. Suppose that $\{x(\zeta)\}$ is an eventually positive solution of (1.1). Then

$$
v(\zeta)>0, \Delta v(\zeta)>0 \text {, and } \Delta\left(\varphi(\zeta)(\Delta v(\zeta))^{\xi}\right) \leq 0 \text {, eventually. }
$$


Proof. By assumption, $v(\zeta)>0$ and we can find an integer $\zeta_{1} \geq \zeta_{0}$ with

$$
x(\zeta)>0, x\left(\kappa_{i}(\zeta)\right)>0, \text { and } x(\eta(\zeta))>0
$$

for all $\zeta \geq \zeta_{1}$ and $i=1,2, \ldots, m$. From (1.1) it follows that

$$
\Delta\left(\varphi(\zeta)(\Delta v(\zeta))^{\xi}\right)=-\mu(\zeta) x^{\nu}(\eta(\zeta)) \leq 0, \quad \zeta \geq \zeta_{1}
$$

Therefore, $\left\{\varphi(\zeta)(\Delta v(\zeta))^{\xi}\right\}$ is nonincreasing for $\zeta \geq \zeta_{1}$. Assume that $\varphi(\zeta)(\Delta v(\zeta))^{\xi}<$ 0 for $\zeta \geq \zeta_{2} \geq \zeta_{1}$. Hence

$$
\varphi(\zeta)(\Delta v(\zeta))^{\xi} \leq \varphi\left(\zeta_{2}\right)\left(\Delta v\left(\zeta_{2}\right)\right)^{\xi} \text { for all } \zeta \geq \zeta_{2},
$$

that is,

$$
\Delta v(\zeta) \leq\left(\frac{\varphi\left(\zeta_{2}\right)}{\varphi(\zeta)}\right)^{\frac{1}{\xi}} \Delta v(\zeta) \text { for } \zeta \geq \zeta_{2}
$$

Taking summation from $\zeta_{2}$ to $\zeta-1$, we have

$$
v(\zeta) \leq v\left(\zeta_{2}\right)+\left(\varphi\left(\zeta_{2}\right)\right)^{\frac{1}{\xi}} \Delta v\left(\zeta_{2}\right)\left(R(\zeta)-R\left(\zeta_{2}\right)\right) \rightarrow-\infty \text { as } \zeta \rightarrow \infty,
$$

which is a contradiction to $v(\zeta)>0$. Therefore $\varphi(\zeta)(\Delta v(\zeta))^{\xi}>0$ for all $\zeta \geq \zeta_{2}$ and hence $\Delta v(\zeta)>0$, and hence the proof.

Lemma 2.3. Suppose that $\{x(\zeta)\}$ is an eventually positive solution of (1.1). Then there exists $\zeta \geq \zeta_{0}$ with

$$
v(\zeta) \geq(\varphi(\zeta))^{\frac{1}{\xi}} \Delta v(\zeta) R\left(\zeta, \zeta_{1}\right), \quad \zeta \geq \zeta_{1}
$$

and

$$
\Delta\left(\frac{v(\zeta)}{R\left(\zeta, \zeta_{1}\right)}\right) \leq 0, \quad \zeta \geq \zeta_{1}
$$

where $R\left(\zeta, \zeta_{1}\right)=R(\zeta)-R\left(\zeta_{1}\right)$.

Proof. Applying procedure as we followed in the proof of Lemma 2.2, we get (2.1) for all $\zeta \geq \zeta_{1} \geq \zeta_{0}$. Since $\left\{\varphi(\zeta)(\Delta v(\zeta))^{\xi}\right\}$ is decreasing, we have

$$
v(\zeta) \geq \sum_{s=\zeta_{1}}^{\zeta-1} \varphi^{\frac{1}{\xi}}(s) \frac{\Delta v(s)}{\varphi^{\frac{1}{\xi}}(s)} \geq \varphi^{\frac{1}{\xi}}(\zeta) \Delta v(\zeta) \sum_{s=\zeta_{1}}^{\zeta-1} \frac{1}{\varphi^{\frac{1}{\xi}}(s)}=\varphi^{\frac{1}{\xi}}(\zeta) \Delta v(\zeta) R\left(\zeta, \zeta_{1}\right) .
$$

Using the above inequality, we have

$$
\Delta\left(\frac{v(\zeta)}{R\left(\zeta, \zeta_{1}\right)}\right)=\frac{\varphi^{\frac{1}{\xi}}(\zeta) \Delta v(\zeta) R\left(\zeta, \zeta_{1}\right)-v(\zeta)}{\varphi^{\frac{1}{\xi}}(\zeta) R\left(\zeta, \zeta_{1}\right) R\left(\zeta+1, \zeta_{2}\right)} \leq 0
$$

We conclude that $\left\{\frac{v(\zeta)}{R\left(\zeta, \zeta_{1}\right)}\right\}$ is decreasing for $\zeta \geq \zeta_{1}$ and hence the proof. 
Lemma 2.4. Suppose that $\{x(\zeta)\}$ is an eventually positive solution of (1.1). Then

$$
x(\zeta) \geq P(\zeta) v(\zeta) \text {, eventually. }
$$

Proof. Lemma 2.2 implies that there exists $\zeta_{1} \geq \zeta_{0}$ such that $v(\zeta)>0$, for all $\zeta \geq \zeta_{1}$.

Now,

$$
\begin{aligned}
x(\zeta) & =v(\zeta)-\sum_{i=1}^{m} p_{i}(\zeta) x^{\lambda_{i}}\left(\kappa_{i}(\zeta)\right) \\
& \geq v(\zeta)-\sum_{i=1}^{m} p_{i}(\zeta) v^{\lambda_{i}}\left(\kappa_{i}(\zeta)\right) \\
& \geq v(\zeta)-\sum_{i=1}^{m} p_{i}(\zeta) v^{\lambda_{i}}(\zeta) .
\end{aligned}
$$

Using Lemma 2.1, we get

$$
\begin{aligned}
x(\zeta) & \geq v(\zeta)-\sum_{i=1}^{m} p_{i}(\zeta)\left(\lambda_{i} v(\zeta)+\left(1-\lambda_{i}\right)\right) \\
& =\left(1-\sum_{i=1}^{m} \lambda_{i} p_{i}(\zeta)\right) v(\zeta)-\sum_{i=1}^{m}\left(1-\lambda_{i}\right) p_{i}(\zeta) .
\end{aligned}
$$

Using the fact that $\{v(\zeta)\}$ is positive increasing sequence, and $\{\psi(\zeta)\}$ is positive decreasing sequence which tends to zero, we find an integer $\zeta_{2} \geq \zeta_{1}$ such that

$$
v(\zeta) \geq \psi(\zeta) \text { for } \zeta \geq \zeta_{2} .
$$

Applying (2.4) in (2.3), we get

$$
x(\zeta) \geq P(\zeta) v(\zeta), \quad \zeta \geq \zeta_{2} .
$$

The proof is now complete.

Lemma 2.5. Suppose that $\{x(\zeta)\}$ is an eventually positive solution of (1.1). Then there exists $\zeta^{*} \geq \zeta_{0}$ and $\theta>0$ such that

$$
0<v(\zeta)<\theta R(\zeta)
$$

and

$$
R\left(\zeta, \zeta^{*}\right)\left[\sum_{s=\zeta}^{\infty} \mu(s) x^{\nu}(\eta(s))\right]^{\frac{1}{\xi}} \leq v(\zeta)
$$

hold for all $\zeta \geq \zeta^{*}$. 
Proof. There exists an integer $\zeta_{1} \geq \zeta_{0}$ with $x(\zeta)>0, x\left(\kappa_{i}(\zeta)\right)>0$, and $x(\eta(\zeta))>0$ for all $\zeta \geq \zeta_{1}$ and for all $i=1,2, \ldots, m$. So there exists a $\zeta_{2} \geq \zeta_{1}$ such that Lemma 2.2 holds and $\{v(\zeta)\}$ satifies (2.1) for $\zeta \geq \zeta_{2}$. From $\varphi(\zeta)(\Delta v(\zeta))^{\xi}>0$ and being nonincreasing, we have

$$
\Delta v(\zeta) \leq\left(\frac{\varphi\left(\zeta_{2}\right)}{\varphi(\zeta)}\right)^{\frac{1}{\xi}} \Delta v\left(\zeta_{2}\right), \quad \zeta \geq \zeta_{2}
$$

Sum the inequality from $\zeta_{2}$ to $\zeta-1$, we obtain

$$
v(\zeta) \leq v\left(\zeta_{2}\right)+\left(\varphi\left(\zeta_{2}\right)\right)^{\frac{1}{\xi}} \Delta v\left(\zeta_{2}\right) R\left(\zeta, \zeta_{2}\right) .
$$

Since $R\left(\zeta, \zeta_{2}\right) \rightarrow \infty$ as $\zeta \rightarrow \infty$ and $\varphi(\zeta)(\Delta v(\zeta))^{\xi}$ is positive and decreasing, there exists a $\theta>0$ and $\zeta^{*} \geq \zeta_{2}$ such that (2.5) holds. On the other hand, $\lim _{\zeta \rightarrow \infty} \varphi(\zeta)(\Delta v(\zeta))^{\xi}$ exists, and summing (1.1) from $\zeta$ to $l-1$ implies

$$
\varphi(l)(\Delta v(l))^{\xi}-\varphi(\zeta)(\Delta v(\zeta))^{\xi}=\sum_{s=\zeta}^{l-1} \mu(s) x^{\nu}(\eta(s)) .
$$

Taking limit $l \rightarrow \infty$, we have

$$
\varphi(\zeta)(\Delta v(\zeta))^{\xi} \geq \sum_{s=\zeta}^{\infty} \mu(s) x^{\nu}(\eta(s))
$$

that is,

$$
\Delta v(\zeta) \geq\left(\frac{1}{\varphi(\zeta)} \sum_{s=\zeta}^{\infty} \mu(s) x^{\nu}(\eta(s))\right)^{\frac{1}{\xi}}
$$

Therefore,

$$
v(\zeta) \geq \sum_{u=\zeta_{1}}^{\zeta-1}\left[\frac{1}{\varphi(u)} \sum_{s=u}^{\infty} \mu(s) x^{\nu}(\eta(s))\right]^{\frac{1}{\xi}} \geq R\left(\zeta, \zeta_{1}\right) \sum_{s=\zeta}^{\infty} \mu(s) x^{\nu}(\eta(s))
$$

and hence the proof.

\section{SUFFICIENT CONDITIONS FOR OSCILLATION}

Theorem 3.1. If

$$
\sum_{\zeta=\zeta_{0}}^{\infty} Q_{1}(\zeta)=\infty
$$

holds, then (1.1) is oscillatory. 
Proof. Assume that $\{x(\zeta)\}$ is an eventually positive solution of (1.1). Then, we can find an integer $\zeta_{1} \geq \zeta_{0}$ with $x(\zeta)>0, x\left(\kappa_{i}(\zeta)\right)>0$, and $x(\eta(\zeta))>0$ for all $\zeta \geq \zeta_{1}$, $i=1,2, \ldots, m$ and Lemmas 2.2 and 2.4 hold for $\zeta \geq \zeta_{1}$. From (1.1), we have

$$
\Delta\left(\varphi(\zeta)(\Delta v(\zeta))^{\xi}\right)+\mu(\zeta) P^{\nu}(\eta(\zeta)) v^{\nu}(\eta(\zeta)) \leq 0
$$

for all $\zeta \geq \zeta_{1}$. Applying (2.1), we conclude that $\lim _{\zeta \rightarrow \infty} \varphi(\zeta)(\Delta v(\zeta))^{\xi}$ exists and there exists an integer $\zeta_{2} \geq \zeta_{1}$ and a positive number $c$ with $v(\zeta) \geq c$ for $\zeta \geq \zeta_{2}$. Summing (3.2) from $\zeta_{2}$ to $\zeta-1$, we have

$$
\left.c^{\nu} \sum_{s=\zeta_{2}}^{\zeta-1} \mu(s) P^{\nu}(\eta(s)) \leq \varphi\left(\zeta_{2}\right)\left(\Delta v\left(\zeta_{2}\right)\right)^{\xi}-\varphi(\zeta)\right)(\Delta v(\zeta))^{\xi}<\infty \text { as } \zeta \rightarrow \infty
$$

which contradicts (3.1).

The proof is similar in the case where $\{x(\zeta)\}$ is an eventually negative solution.

Remark 3.2. Theorem 3.1 is true for any $\nu$ and $\xi$.

Now, we derive criteria for oscillation to (1.1) when $\nu>1$.

\section{Theorem 3.3. If}

$$
\sum_{\zeta=\zeta_{0}}^{\infty} Q_{2}(\zeta)=\infty
$$

holds, then (1.1) is oscillatory.

Proof. Using the procedure followed in the Theorem 3.1, we get (3.2). Using (2.4) in (3.2), we see that

$$
\Delta\left(\varphi(\zeta)(\Delta v(\zeta))^{\xi}\right)+\mu(\zeta) P^{\nu}(\eta(\zeta)) \psi^{\nu-1}(\eta(\zeta)) v(\eta(\zeta)) \leq 0
$$

The remainder of the proof is identical to that of Theorem 3.1, thus proved.

Next, we derive an oscillation criteria for the equation (1.1) where $0<\nu<1$.

Theorem 3.4. If

$$
\sum_{\zeta=\zeta_{0}}^{\infty} Q_{3}(\zeta)=\infty
$$

holds, then (1.1) is oscillatory. 
Proof. We arrived (3.2) by using the process followed in the proof of Theorem 3.1. From (3.2), we have

$$
\Delta\left(\varphi(\zeta)(\Delta v(\zeta))^{\xi}\right)+\mu(\zeta) P^{\nu}(\eta(\zeta)) R^{\nu-1}\left(\left(\eta(\zeta), \zeta_{1}\right) \frac{v^{\nu-1}(\eta(\zeta))}{R^{\nu-1}\left(\eta(\zeta), \zeta_{1}\right)} \leq 0\right.
$$

for $\zeta \geq \zeta_{2} \geq \zeta_{1}$. Since $\left\{\frac{v(\zeta)}{R\left(\zeta, \zeta_{1}\right)}\right\}$ is a non-increasing sequence, there is a constant $\beta$ such that

$$
\frac{v(\eta(\zeta))}{R\left(\eta(\zeta), \zeta_{1}\right)} \leq \beta \text { for } \zeta \geq \zeta_{2}
$$

Using (3.7) and $\nu<1$ in (3.6), we have

$$
\Delta\left(\varphi(\zeta)(\Delta v(\zeta))^{\xi}\right)+\frac{\mu(\zeta) P^{\nu}(\eta(\zeta)) R^{\nu-1}\left(\eta(\zeta), \zeta_{1}\right)}{\beta^{\nu-1}} v(\eta(\zeta)) \leq 0 .
$$

The remainder of the part is same to Theorem 3.3, thus proved.

In the next theorem, we suppose that there is a constant $\nu_{1}$, the ratio of odd positive integers with $0<\nu<\nu_{1}<\xi$.

Theorem 3.5. If

$$
\sum_{\zeta=\zeta_{0}}^{\infty} Q_{4}(\zeta)=\infty
$$

holds, then (1.1) is oscillatory.

Proof. Suppose that $\{x(\zeta)\}$ is an eventually positive solution of (1.1). Now, we can find an integer $\zeta_{1} \geq \zeta_{0}$ with $x(\zeta)>0, x\left(\kappa_{i}(\zeta)\right)>0, i=1,2, \ldots, m, x(\eta(\zeta))>0$ for all $\zeta \geq \zeta_{1}$, and Lemmas 2.2 and 2.5 hold for $\zeta \geq \zeta_{1}$. Hence,

$$
v(\zeta) \geq R\left(\zeta, \zeta_{1}\right) U^{\frac{1}{\xi}}(\zeta) \geq 0 \text { for } \zeta \geq \zeta_{1}
$$

Using (2.2), (2.5), $\nu-\nu_{1}<0$, and (3.9), we have

$$
\begin{aligned}
x^{\nu}(\zeta) & \geq P^{\nu}(\zeta) v^{\nu-\nu_{1}}(\zeta) v^{\nu_{1}}(\zeta) \\
& \geq P^{\nu}(\zeta)\left(\theta R\left(\zeta, \zeta_{1}\right)\right)^{\nu-\nu_{1}} v^{\nu_{1}}(\zeta) \\
& \geq P^{\nu}(\zeta)\left(\theta R\left(\zeta, \zeta_{1}\right)\right)^{\nu-\nu_{1}} R^{\nu_{1}}\left(\zeta, \zeta_{1}\right) U^{\frac{\nu_{1}}{\xi}}(\zeta) \\
& =P^{\nu}(\zeta) \theta^{\nu-\nu_{1}} R^{\nu}\left(\zeta, \zeta_{1}\right) U^{\frac{\nu_{1}}{\xi}}(\zeta) \text { for } \zeta \geq \zeta_{2} .
\end{aligned}
$$


Since $\Delta U(\zeta)=-\mu(\zeta) x^{\nu}(\eta(\zeta)) \leq 0, \quad \zeta \geq \zeta_{2}$, that is $\{U(\zeta)\}$ is a non-increasing sequence, then

$$
\begin{aligned}
x^{\nu}(\eta(\zeta)) & \geq P^{\nu}(\eta(\zeta)) \theta^{\nu-\nu_{1}} R^{\nu}\left(\eta(\zeta), \zeta_{1}\right) U^{\frac{\nu_{1}}{\xi}}(\eta(\zeta)) \\
& \geq P^{\nu}(\eta(\zeta)) \theta^{\nu-\nu_{1}} R^{\nu}\left(\eta(\zeta), \zeta_{1}\right) U^{\frac{\nu_{1}}{\xi}}(\zeta) .
\end{aligned}
$$

Therefore,

$$
\Delta\left(U^{1-\frac{\nu_{1}}{\xi}}\right) \leq\left(1-\frac{\nu_{1}}{\xi}\right) U^{-\frac{\nu_{1}}{\xi}}(\zeta) \Delta U(\zeta)
$$

Summing (3.11) from $\zeta_{2}$ to $\zeta-1$ and from $U(\zeta)>0$, we have

$$
\begin{aligned}
\infty>U^{1-\frac{\nu_{1}}{\xi}}\left(\zeta_{2}\right) & \geq\left(1-\frac{\nu_{1}}{\xi}\right)\left[-\sum_{s=\zeta_{2}}^{\zeta-1} U^{-\frac{\nu_{1}}{\xi}}(s) \Delta U(s)\right] \\
& =\left(1-\frac{\nu_{1}}{\xi}\right) \sum_{s=\zeta_{2}}^{\zeta-1} U^{-\frac{\nu_{1}}{\xi}}(s) \mu(s) x^{\nu}(\eta(s)) \\
& \geq \frac{\left(1-\frac{\nu_{1}}{\xi}\right)}{\theta^{\left(\nu_{1}-\nu\right)}} \sum_{s=\zeta_{2}}^{\zeta-1} \mu(s) P^{\nu}(\eta(s)) R^{\nu}\left(\eta(s), \zeta_{1}\right)
\end{aligned}
$$

which contradicts (3.8) as $\zeta \rightarrow \infty$ and hence proved.

In the next theorem, we suppose that there is a constant $\nu_{2}$, the ratio of odd positive integers with $\lambda<\nu_{2}<\nu$.

Theorem 3.6. Assume that $\Delta \varphi(\zeta) \geq 0$. Suppose that

$$
\sum_{\zeta=\zeta_{0}}^{\infty}\left[\frac{1}{\varphi(\eta(\zeta))} \sum_{s=\zeta+1}^{\infty} Q_{1}(s)\right]^{\frac{1}{\xi}}=\infty
$$

Then (1.1) is oscillatory.

Proof. Assume that $\{x(\zeta)\}$ is an eventually positive solution of (1.1). Then, we can find an integer $\zeta_{1} \geq \zeta_{0}$ with $x(\zeta)>0, x\left(\kappa_{i}(\zeta)\right)>0,(i=1,2, \ldots, m), x(\eta(\zeta))>0$ and Lemmas 2.2 and 2.4 hold for $\zeta \geq \zeta_{1}$. We conclude that $\{v(\zeta)\}$ satisfies (2.1), $\{v(\zeta)\}$ is increasing and $x(\zeta) \geq P(\zeta) v(\zeta)$ for all $\zeta \geq \zeta_{1}$. So,

$$
x^{\nu}(\zeta) \geq P^{\nu}(\zeta) v^{\nu}(\zeta) \geq P^{\nu}(\zeta) v^{\nu-\nu_{2}}(\zeta) v^{\nu_{2}}(\zeta) \geq P^{\nu}(\zeta) v^{\nu-\nu_{2}}\left(\zeta_{1}\right) v^{\nu_{2}}(\zeta)
$$

implies that

$$
x^{\nu}(\eta(\zeta)) \geq P^{\nu}(\eta(\zeta)) v^{\nu-\nu_{2}}\left(\zeta_{1}\right) v^{\nu_{2}}(\eta(\zeta)) \text { for } \zeta \geq \zeta_{2} \geq \zeta_{1}
$$


Using (2.7) and (3.13), we have

$$
\varphi(\zeta)(\Delta v(\zeta))^{\xi} \geq v^{\nu-\nu_{2}}\left(\zeta_{1}\right)\left[\sum_{s=\zeta}^{\infty} \mu(s) P^{\nu}(\eta(\zeta))\right] v^{\nu_{2}}(\eta(\zeta))
$$

for $\zeta \geq \zeta_{2}$. From $\left\{\varphi(\zeta)(\Delta v(\zeta))^{\xi}\right\}$ being nonincreasing and $\eta(\zeta) \leq \zeta$, we have

$$
\varphi(\eta(\zeta))(\Delta v(\eta(\zeta)))^{\frac{1}{\xi}} \geq \varphi(\zeta)(\Delta v(\zeta))^{\frac{1}{\xi}}
$$

Using the increasing nature of $\{v(\zeta)\}$ and the decreasing nature of $\left\{\varphi(\zeta)(\Delta v(\zeta))^{\xi}\right\}$ in (3.14)

$$
\varphi(\zeta)(\Delta v(\eta(\zeta)))^{\xi} \geq v^{\nu-\nu_{2}}\left(\zeta_{1}\right)\left[\sum_{s=\zeta+1}^{\infty} \mu(s) P^{\nu}(\eta(\zeta))\right] v^{\nu_{2}}(\eta(\zeta+1))
$$

or

$$
\frac{\Delta v(\eta(\zeta))}{v^{\frac{\nu_{2}}{\xi}}(\eta(\zeta+1))} \geq\left[\frac{v^{\nu-\nu_{2}}\left(\zeta_{1}\right)}{\varphi(\eta(\zeta))} \sum_{s=\zeta+1}^{\infty} \mu(s) P^{\nu}(\eta(s))\right]^{\frac{1}{\xi}}
$$

for $\zeta \geq \zeta_{2}$.

Sum the above inequality from $\zeta_{2}$ to $\zeta-1$, we get

$$
\sum_{u=\zeta_{2}}^{\zeta-1} \frac{\Delta v(\eta(u))}{v^{\frac{\nu_{2}}{\xi}}(\eta(u+1))} \geq v^{\nu-\nu_{2}}\left(\zeta_{1}\right) \sum_{u=\zeta_{2}}^{\zeta-1}\left[\frac{1}{\varphi(\eta(u))} \sum_{s=u+1}^{\infty} \mu(s) P^{\nu}(\eta(s))\right]^{\frac{1}{\xi}}, \text { for } \zeta \geq \zeta_{2} .
$$

This implies that

$$
\frac{1}{1-\frac{\nu_{2}}{\xi}}\left[\frac{1}{v^{\frac{\nu_{2}}{\xi}-1}(\eta(\zeta))}-\frac{1}{v^{\frac{\nu_{2}}{\xi}-1}\left(\eta\left(\zeta_{2}\right)\right)}\right] \geq v^{\frac{\nu-\nu_{2}}{\xi}}\left(\zeta_{1}\right) \sum_{u=\zeta_{2}}^{\zeta-1}\left[\frac{1}{\varphi(\eta(u))} \sum_{s=u+1}^{\infty} \mu(s) P^{\nu}(\eta(s))\right]^{\frac{1}{\xi}} .
$$

Since $\xi<\nu_{2}$, we have

$$
\frac{1}{\frac{\nu_{2}}{\xi}-1} v^{1-\frac{\nu_{2}}{\xi}}\left(\eta\left(\zeta_{2}\right)\right) \geq v^{\frac{\nu-\nu_{2}}{\xi}}\left(\zeta_{1}\right) \sum_{u=\zeta_{2}}^{\zeta-1}\left[\frac{1}{\varphi(\eta(u))} \sum_{s=u+1}^{\infty} \mu(s) P^{\nu}(\eta(s))\right]^{\frac{1}{\xi}}
$$

which implies that

$$
\sum_{u=\zeta_{2}}^{\zeta-1}\left[\frac{1}{\varphi(\eta(u))} \sum_{s=u+1}^{\infty} \mu(s) P^{\nu}(\eta(s))\right]^{\frac{1}{\xi}}<\infty .
$$

This contradicts (3.12) as $\zeta \rightarrow \infty$. This contradiction shows that $\{x(\zeta)\}$ cannot be an eventually positive solution. We omit the case where $\{x(\zeta)\}$ is eventually negative solution.

Finally, we offer some instances to illustrate the usefulness and viability of our main results. 


\section{EXAMPLE}

Example 4.1. Consider the second-order difference equation

$$
\Delta\left(\zeta\left(\Delta\left(x(\zeta)+\frac{1}{\zeta} x^{\frac{1}{3}}(\zeta-1)+\frac{1}{\zeta^{2}} x^{\frac{1}{5}}(\zeta-2)\right)\right)^{3}\right)+\zeta^{6} x^{5}(\zeta-1)=0 ; \quad \zeta \geq 2
$$

where $\varphi(\zeta)=\zeta, \mu(\zeta)=\zeta^{6}, \eta(\zeta)=\zeta-1, \kappa_{1}(\zeta)=\zeta-1, \kappa_{2}(\zeta)=\zeta-2, p_{1}(\zeta)=\frac{1}{\zeta}$, $p_{2}(\zeta)=\frac{1}{\zeta^{2}}$. Take $\psi(\zeta)=\frac{1}{\zeta^{6}}$. Now, we can easily verify all assumptions of the Theorem 3.1. Then by Theorem 3.1, (3.1) is oscillatory.

Example 4.2. Let us investigate the second-order difference equation

$\Delta\left(\frac{1}{\zeta^{\frac{1}{5}}}\left(\Delta\left(x(\zeta)+\frac{1}{\zeta} x^{\frac{1}{3}}(\zeta-1)+\frac{1}{\zeta^{2}} x^{\frac{1}{5}}(\zeta-2)\right)\right)^{\frac{1}{5}}\right)+\zeta^{\frac{2}{5}} x^{\frac{1}{3}}(\zeta-2)=0 ; \quad \zeta \geq 2$.

Here, we have $\varphi(\zeta)=\frac{1}{\zeta^{\frac{1}{5}}}, \mu(\zeta)=\zeta^{\frac{2}{5}}, p_{1}(\zeta)=\frac{1}{\zeta}, p_{2}(\zeta)=\frac{1}{\zeta^{2}}, \lambda_{1}=\frac{1}{3}, \lambda_{2}=\frac{1}{5}, \xi=\frac{1}{5}$, $\nu=\frac{1}{3}, \kappa_{1}(\zeta)=\zeta-1, \kappa_{2}(\zeta)=\zeta-2$ and $\eta(\zeta)=\zeta-2$. Set $\psi(\zeta)=\frac{1}{\zeta}$. We can easily verify all assumption of the Theorem 3.4. Hence, by Theorem 3.4, (3.2) is oscillatory.

\section{CONCLUSION}

We explored the oscillatory behavior of solutions to (1.1) in this study and derived various sufficient conditions for oscillation to (1.1). Examples are given to demonstrate the importance of our results.

\section{REFERENCES}

[1] R. P. Agarwal, Difference Equations and Inequalities, Theory, Methods and Applications. CRC Press. ISBN: 9780824790073, (2000).

[2] R. P. Agarwal, M. Bohner, S. R. Grace and D. O'Regan, Discrete Oscillation Theory, Hindawi Publishing Corporation, New York, (2005).

[3] R. P. Agarwal, S. R. Grace and D. O’ Regan, Oscillation Theory for Difference and Functional Differential Equations, Kluwer Academic, Dordrecht, (2000).

[4] R. P. Agarwal, S. R. Grace, and D. O'Regan, Nonoscillatory solutions for discrete equations, Computeres and Mathematics with Applications, 45(6-9)(2003),12971302.

[5] A. G. Balanov, N. B. Janson, P. V. E. McClintock, R. W. Tucks and C.H. T. Wang, Bifurcation analysis of a neutral delay differential equation modelling the torsional motion of driven drill-string, Chaos, Solitons and Fractals, 15(2003), 381-394. 
[6] A. Bellen, N. Guglielmi and A. E. Ruchli, Methods for linear systems of circuit delay differential equations of neutral type, IEEE Trans. Circ. Syst - I, 46(1999), 212-216.

[7] J. Cheng, Existence of a nonoscillatory solution of a second-order linear neutral difference equations, Applied Mathematics Letters, 20(8)(2007), 892-899.

[8] J. Cheng and Y. Chu, Oscillation theroem for second-order difference equations, Taiwanese Journal of Mathematics, 12(3)(2008), 623-633.

[9] S. S. Cheng and W. T. Patula, An existence theorem for a nonlinear difference equations, Nonlinear Analysis: Theory, Methods and Applications, 20(3)(1993), 193-203.

[10] C. Dharuman, J. R. Graef, E. Thandapani and K. S. Vidhyaa, Oscillation of second order difference equation with a sub-linear neutral term, J. Math. Appl., 40(2017), 59-67.

[11] L. H. Erbe, Q. K. Kong and B. G. Zhang, Oscillation Theory for Functional Difference Equations, Marcel Dekker, New york, USA, (1995).

[12] P. Gopalakrishnan, A. Murugesan and C. Jayakumar, Oscillation conditions of the second order noncanonical difference equations, J. Math. Computer Sci., 25(2021), 351-360.

[13] P. Gopalakrishnan, A. Murugesan, Dafik and Ika Hesti Agustin, Oscillation and Asymptotic Behavior of Second-Order Half-Linear Noncanonical Difference Equations of Advanced Type, Journal of Physics: Conference Series IOP Science (Accepted).

[14] K. Gopalsamy, Stability and Oscillations in Population Dynamics, Kluwer Acad. Pub, Bostan, (1992).

[15] I. Gyori and G. Ladas, Oscillation Theory of Delay Differential Equations with Applications, Clarendon press, Ox-ford, (1991).

[16] J. K. Hale, Theory of Functional Differential Equations, 2nd edn, Applied Mathematical Sciences, Vol.3. Springer, New York, (1997).

[17] Z. Liu, Y. Xu, and S. M. Kang, Global solvability for a second order nonlinear neutral delay difference equations, Computers and Mathematics with Applications, 57(4)(2009), 587-595.

[18] J. W. Luo and D. D. Bainov, Oscillatory and asymptotic behavior of second-order neutral difference equations with maxima,J. Math. Appl., 131(1-2)(2001), 333341.

[19] R. E. Mickens, Difference Equations, Theory and Applications, Van NostrandReinhold, New York, (1990). 
[20] M. Migda and J. Migda, Asymptotic properties of solutions of secondorder neutral difference equation, Nonlinear Analysis: Theory, Methods and Applications,63(5-7)(2005), 789-799.

[21] A. Murugesan and C. Jayakumar, Oscillation condition for second order halflinear advanced difference equation with variable coefficients, Malaya Journal of Mathematik, 8(4)(2020), 1872-1879.

[22] C. J. Tian and S. S. Cheng, Oscillation criteria for delay neutral difference equations with positive and negative coefficients, Bul. Soc. parana Math. 21(2003), 1-12.

[23] W. Xiong and J. Liang, Novel stability criteria for neutral systems with multiple time delays, Chaos, Solitons and Fractals, 32(2007), 1735-1741.

[24] J. Zhou, T. Chen and L. Xiang, Robust synchronization of delayed neutral networks based on adaptive control and parameters identification, Chaos, Solitons and Fractals, 27(2006), 905-913. 
